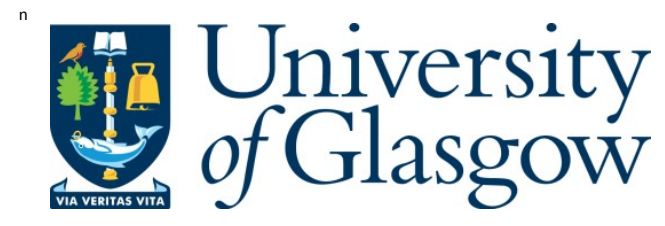

Vinciarelli, A ., Salamin, H., and Polychroniou, A . (2014) Negotiating over mobile phones: calling or being called can make the difference.Cognitive Computation, 6 (4). pp. 677-688. ISSN 1866-9956

Copyright (c) 2014 Springer

A copy can be downloaded for personal non-commercial research or study, without prior permission or charge

Content must not be changed in any way or reproduced in any format or medium without the formal permission of the copyright holder(s)

http://eprints.gla.ac.uk/100489/

Deposited on: 18 December 2014

Enlighten - Research publications by members of the University of Glasgow http://eprints.gla.ac.uk 


\title{
Negotiating Over Mobile Phones: Calling or Being Called can Make the Difference
}

\author{
Alessandro Vinciarelli · Hugues Salamin · Anna Polychroniou
}

Received: date / Accepted: date

\begin{abstract}
Mobile phones pervade our everyday life like no other technology, but the effects they have on one-to-one conversations is still relatively unknown. This paper focuses on how mobile phones influence negotiations, i.e. on discussions where two parties try to reach an agreement starting from opposing preferences. The experiments involve 60 pairs of unacquainted individuals (120 subjects). They must make a "yes" or "no" decision on whether several objects increase the chances of survival in a polar environment or not. When the participants disagree about a given object (one says "yes" and the other says "no"), they must try to convince one another and reach a common decision. Since the subjects discuss via phone, one of them (selected randomly) calls while the other is called. The results show that the caller convinces the receiver in $70 \%$ of the cases ( $p$-value $=0.005$ according to two-tailed a binomial test). Gender, age, personality and conflict handling style, measured during the experiment, fail in explaining such a persuasiveness difference. Calling or being called appears to be the most important factor behind the observed result.
\end{abstract}

Keywords Negotiation; Persuasiveness; Mobile Phones, Personality, Conflict Handling Style

\section{Introduction}

No other technology penetrated our everyday life as quickly and ubiquitously as mobile phones. A mere 15 years on the consumer electronics market were sufficient to have one mobile phone subscription for every third person in the world, with 82 subscriptions per 100 persons in Europe (the most

A. Vinciarelli ${ }^{1,2} \cdot$ H. Salamin ${ }^{1} \cdot$ A.Polychroniou ${ }^{1}$

${ }^{1}$ School of Computing Science, University of Glasgow, UK

${ }^{2}$ Idiap Research Institute, Switzerland

E-mail: \{vincia,hasalamin,annap\}@dcs.gla.ac.uk "mobile" continent) and 19 countries where the number of subscriptions exceeded the size of the population (see [20, 24] for up-to-date figures). The impact of such a widespread diffusion on social interactions was extensively investigated at the scale of large social networks [27, 28], but surprisingly little work was done to understand the effect, if any, on one-to-one conversations, one of the scenarios where mobile phones are most commonly used [4].

In particular, mobile phones were adopted extensively to analyze everyday social phenomena because they are equipped to sense and measure behavior with unprecedented depth and precision [38]. However, it is not clear whether mobile phones can be considered as passive communication media or they change our way of engaging in social interactions [13, 27], our communication practices [19] and our social behavior during one-to-one conversations [4]. In other words, to the best of our knowledge, it is not clear whether mobile phones modify the very phenomena they are supposed to allow.

This article tries to fill, at least partially, such a gap and shows experiments where 60 pairs of individuals (120 subjects in total) perform a negotiation task talking via standard mobile phones. Negotiation is a process "for resolving opposing preferences [...] with the goal of reaching agreement" [9]. It is a common phenomenon - it can be observed from playgrounds to international relations - and the literature proposes several works aimed at assessing the impact of communication media on its outcomes [6]. The reason is that the diffusion of communication technologies has been consistently increasing in the last three decades. Therefore, more and more decisions are made in technology-mediated interactions [45]. Furthermore, it was shown that the communication media "[affect] our definition of the negotiation game and the behavior deemed appropriate for the interaction" [6]. 
In the experiments of this work, the participants must decide whether the items of a predefined list (see Section 3) increase the chances of survival after crashing with a plane in a polar area (the Winter Survival Task, WST). First, the 120 subjects perform the task individually and make a "yes" or "no" decision for every item in the list. Then, each subject participates in a phone call with another participant. If the two subjects involved in the same call have made a different decision about the same item (one says "yes" and the other says "no"), they have to negotiate a common answer. In other words, if the two call participants have opposite opinions about the same item, one of the two must convince the other to change mind.

The subjects of each call are unacquainted, do not know anything about one another, receive the same information and fill the same questionnaires. The only, inevitable difference in each dyad is that one of the subjects, selected randomly, is instructed to call while the other is instructed to wait for being called. The results show that the subjects being called manage to persuade their counterparts $70 \%$ of the time ( $p$-value $=0.005$ according to a two-tailed binomial test). In other words, calling or being called seems to influence, to a significant extent, the outcome of the negotiations about a common answer.

In line with research on negotiation (see [6] for an extensive survey), such a result seems to suggest that the mere use of phones results into a subtle, but effective form of Behavior Change Technique $[2,10]$ known as social psychological intervention [1]: “Altering people's views of themselves, or how they think others view them, can lead to cascading changes in motivation and performance" [49]. Gender, age, personality and conflict handling style, measured during the experiment, were considered as alternative explanations, but fail in accounting for the observed persuasiveness differences. In other words, calling or being called seems to be one of the most important factors behind the effectiveness of an individual in convincing others.

The rest of this paper is organized as follows: Section 2 presents previous work on the interplay between persuasiveness, negotiation and communication technologies, Section 3 describes experimental protocol and data, Section 4 reports on the results and the final Section 5 draws some conclusions.

\section{Previous Work}

This section surveys previous work on the relationship between communication media and negotiation (see Section 2.1), effect of personal characteristics on negotiation outcomes (see Section 2.2) and mobile technologies aimed at influencing the behavior of people (see Section 2.3).

\subsection{Technology Mediated Negotiation}

The main constructs underlying the effect of communication media on negotiations are social awareness ("the degree of consciousness and attention to the other(s) in a social interaction" [30]) and richness ("feedback capability, communication channels utilized, language variety and personal focus" [45]). Therefore, several works investigate how negotiation outcomes change when using communication media of increasing richness [37, 43, 45, 47]. This latter cannot be measured quantitatively, but there is consensus on the following ordering (from lowest to highest richness): handwritten messages, e-mail, chat, telephone, video-conference and face-to-face interactions.

The experiments of [43] involve 55 dyads (110 subjects in total) acting in a buyer-seller scenario where the goal is to find an agreement about the price of several commodities. Some dyads negotiate face-to-face (with and without a computer at disposition), others via telephone or chat. The results show that the joint profit increases when moving from a purely textual (chat) to a verbal communication medium (the phone), but it does not increase further when adding the visual channel (face-to-face). Furthermore, adding the visual channel is an advantage only if both negotiators are cooperative, i.e. they try to maximize the joint profit of the dyad. The proposed explanation is that the possibility of speaking reduces the uncertainty about the task (hence the increase in joint profit when adding the verbal channel) while the possibility of watching one another increases only the awareness of the orientation of the counterpart (hence the absence of an effect on the joint profit when there are non-cooperative negotiators).

Media richness appears to have an effect in [37] as well. The experiments of this work involve 150 subjects bargaining with one another via four different media (face-to-face, videoconference, telephone, chat). The results show that the richer the medium, the highest the tendency to collaborate and avoid competition. Similar results are reported in [47], where 166 subjects participated in a bargaining game with asymmetric information. In the experiments, the subjects possessing more information were more likely to keep it secret in low-richness media (often resulting in major advantages) than in high-richness ones. The explanation proposed in both [37, 47] is that higher richness, in particular the inclusion of a video channel, makes it easier to detect noncooperative strategies and adopt countermeasures.

While all experiments above seem to confirm that media richness plays an important role in determining the negotiation outcomes, the results obtained in [45] contradict such a finding. In this work, 316 subjects were involved in a zero-sum game to be performed face-to-face as well as via telephone and videoconference. The results show that the communication medium has no effect on both quality of the 
decisions and time needed to achieve them. However, a possible explanation is that the findings have been obtained in a non-Western setting (South Korea) and it is not possible to exclude cultural differences.

The results above suggest that the phone allows one to access sufficient information about the content of the negotiation (there are no breakdowns like in the case of chat and e-mail), but hides attitude and behavioral strategy of the counterpart. As a result, it is easier for non-cooperative negotiators to maximize their individual profit. The findings surveyed in this section have been obtained with landline phones, but they are likely to apply to mobile phones as well because the richness of the two media is the same. Hence, it can be expected that maximizing individual profit should be easier over mobile phones as well.

\subsection{Negotiation and Interpersonal Relationships}

Since negotiation is an inherently social activity - it cannot take place unless there are at least two parties - several investigations were dedicated to the effect of interpersonal factors, i.e. to "the ways that negotiators' behavior and outcomes depend upon the presence of the other party or parties [...] and the dyadic aspects of negotiation behavior" [46]. This section considers in particular works on the effect of gender, personality and conflict handling style, three factors analyzed in this work as well.

Power differences between negotiators, real or perceived, were the subject of [29]. In the experiments of such work, 38 participants acting in a bargaining scenario, people in a higher power position were shown to have higher propensity to initiate a negotiation. Furthermore, other experiments presented in the same work (involving 62 subjects), show that the tendency to make the first move is beneficial from a bargaining point of view. Hence, negotiating with lower power counterparts appears to be an advantage. The perception of power differences seems to explain some observed gender effects as well (negotiators tend to accept the stereotypical view of women possessing less power than men) [25]. However, extensive meta-analyses contradict such a result and show that gender, overall, has a limited effect on negotiation outcomes [44, 48].

The analysis of [5] focused on negotiators' Big-Five personality traits, in particular Extraversion, Agreeableness and Conscientiousness (see [40] and Section 4.3 for more details). The experiments involved 184 dyads (386 subjects in total) working on a distributive bargaining task, i.e. on the distribution of a finite amount of resources across the dyad members. The results show that higher Extraversion and Agreeableness tend to be associated to lower gains, i.e to lower effectiveness in maximizing personal advantages. Furthermore, Conscientiousness appears to have no significant effect.
The way participants frame the interaction with the other parties is one of the main factors influencing negotiation outcomes [6]. The experiments of [36], performed over 75 dyads (150 subjects in total), show that the outcomes tend to be less satisfactory for people that frame negotiations in terms of relationship (focus on interpesonal concerns) and winning (focus on maximization of personal profit). Viceversa, negotiation outcomes tend to be more favorable for individuals framing their interactions in terms of task (focus on material aspects of the negotiation) and cooperation (focus on maximization of joint profit). The effect of conflict handling style (see [39] and Section 4.4 for more details) was investigated in [11]. The analysis of questionnaires gathered from 70 construction professionals indicate that adopting an integrating conflict style (tendency to maximize joint statisfaction of all parties) tends to achieve functional outcomes, while the adoption of a compromising style (tendency to find a trade-off between conflicting outcomes) is helpful to resolve disputes.

The findings above concern face-to-face negotiations and do not take into account the effect of the communication media. According to the works surveyed in Section 2.1, the phones tend to hide attitude and behavior of negotiation participants. Hence, it can be expected that personal characteristics like personality and conflict handling style, typically manifested through attitude and behavior, should not have a major influence on phone mediated negotiation outcomes.

\subsection{Technology and Behavior Change}

Users react to technologies displaying human-like behavior as if these were actually human [33]. The reason is that unconscious cognitive processes evolved in absence of technology and, therefore, cannot distinguish between natural and artificial human behavior [34]. The phenomenon, known as "Media Equation" [41], is the basis for the development of "persuasive technologies" [15], i.e. machines that aim at changing beliefs, attitude and behavior of their users towards a desired, predefined direction.

Mobile phones, with their ubiquitous presence in everyday life, are an ideal platform for persuasive technologies [26]. Several works (e.g., [3, 12, 18]), show that mobile applications can help people to adopt healthier lifestyles not only by suggesting health oriented practices, but also by supporting social pressure mechanisms, one of the main techniques psychologists adopt to foster behavior change [2]. In [3], experiments involving 130 subjects show that people can be persuaded to perform more physical activity if other subjects, connected through a mobile application, pay for their lack of movement. In [12], mobile phones were used to measure the water intake of 16 subjects and to perform a game where drinking more water allows one to achieve better scores. Furthermore, the same mobile phones support the 
competition with other players towards better water drinking practices. The results show that the subjects actually drink more water especially when the game is social, i.e. it includes competition with others. In the same vein, the experiments of [18] show that mobile applications help 40 people to adopt healthy nutrition styles through social facilitation supported via the phones.

Other works, focused on the way communication technologies change the interaction between people [13, 19, 27] and modify, to a measurable extent, interaction outcomes [7, $8,32]$. The results of [7] show that people collaborating at distance via different communication technologies (e.g., videoconferencing and Instant Messaging) can be persuaded more easily when they think that their interlocutor is geographically closer. In the case of [32], the experiments show that people are more persuasive when they communicate via video than when they do it via audio or text. In the case of [8], the experiments show that political propaganda does not persuade electors when these are reached via phone.

The findings surveyed in this section illustrate the interplay beteween persuasiveness and technology, whether this means to produce persuasive artifacts or to change the persuasiveness of people that adopt technology to interact. The experiments of this work focus on the latter aspect and it is possible to expect that the very use of phones produces changes in persuasiveness.

\section{The Experiment}

The experiment presented in this work is based on the "Winter Survival Task" (WST), a scenario where participants receive a list of 12 items (steel wool, axe, pistol, butter can, newspaper, lighter without fuel, clothing, canvas, airmap, whisky, compass, chocolate) and must identify those that can actually be useful after surviving a plane crash in Northern Canada (in the middle of the winter). The task is frequently used in behavior experiments because average people are unlikely to have experienced a plane crash or to be knowledgeable in survival techniques. Therefore, interaction outcomes result mostly from social, communicative and psychological phenomena and not from competences and skills that participants hold before and independently of the experiment. This is the reason why the analysis does not take into account what people say, but only personal characteristics of the subjects. The scenario was used by NASA to evaluate candidate astronauts [35] and by researchers to, e.g., investigate the interpersonal cohesion in a group [42], help students to develop strategic skills [22], measure group performance [14] or compare the performance of groups and individuals [31].

Overall, the task was performed by 60 pairs of fully unacquainted subjects that have never met before the experiment (120 subjects in total). Table 1 shows gender, national-

\begin{tabular}{llr}
\hline Feature & Description & Distribution \\
\hline Gender & Female & $63(52.5 \%)$ \\
& Male & $57(47.5 \%)$ \\
\hline Background & BSc \& MSc Students & $81(67.5 \%)$ \\
& PhD Students \& Faculties & $23(19.2 \%)$ \\
& Externals & $16(13.3 \%)$ \\
\hline Nationality & British & $118(98.4 \%)$ \\
& American & $1(0.8 \%)$ \\
& Cypriot & $1(0.8 \%)$ \\
\hline
\end{tabular}

Table 1 The subjects. The table shows the distribution in terms of gender, professional background and nationality.

ity and background distribution across the participants. The subjects earn three British Pounds each time they select a correct item (there is a gold standard for the task), but lose the same amount of money when they select a wrong one. In this way, whenever the subjects disagree about a certain item, they are motivated to persuade their interlocutor. Appendix A shows the document given to the participants before starting the experiment.

\subsection{The Participants}

Besides the personal characteristics considered in Section 2.2, other factors can influence the outcome of a negotiation (the following list is not exhaustive): culture, education, power differences, history and nature of previous relationships, etc. (the list is not exhaustive). However, the subjects involved in this work were selected to limit as much as possible the influence of these aspects (see Table 1).

Most of the subjects (118 out of 120) have a British passport and this should limit to a large extent cultural differences. Furthermore, the participants were selected from a pool where all subjects receive or have received a University education (in 104 cases out of 120 they are students or faculties of the School of Psychology at the University of Glasgow). This is expected to reduce the effect of background differences. For what concerns power distances and pre-existing relationships, it was ensured that the subjects involved in each call were fully unacquainted. Hence, they were unaware of possible status differences and did not have any previous contact.

A last important factor in negotiations is the physical setting. For this reason, the participants of each call were asked to sit in two standard, similar offices of the University of Glasgow (the same two offices for all calls). Moreover, the caller was assigned to one of the two offices in the first 30 calls and to the other one in the last 30 ones. In this way, the effect of possible differences between the two offices were distributed evenly across callers and receivers. 


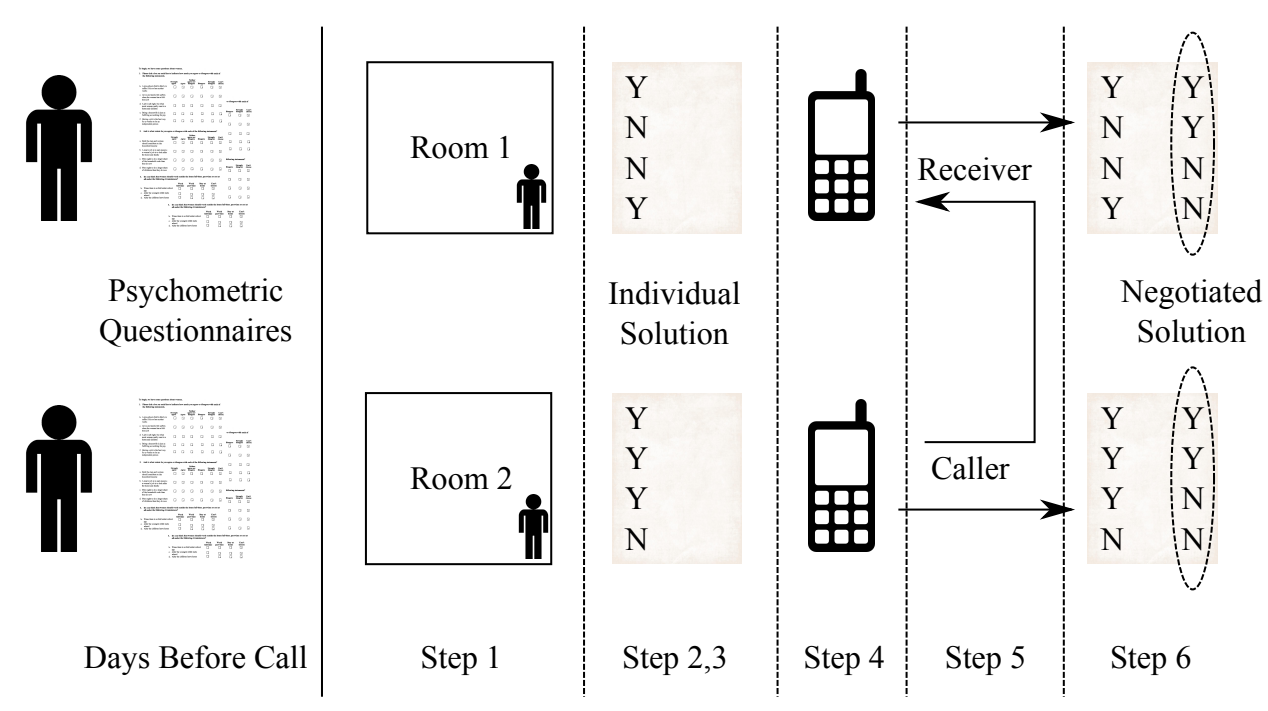

Fig. 1 The picture shows the experimental protocol. The subjects fill self-assessment questionnaires in the days before the call. The day of the call, they sit in one of the two offices used for the experiment (step 1), they read the protocol (step 2), they address the WST task individually (step 3 ), they receive a mobile phone (step 4), they negotiate a common solution during a call (step 5), they deliver a negotiated solution (step 6).

\subsection{Experimental Protocol: Before the Call}

In the days before the experiment, the subjects are asked to fill two self-assessment questionnaires via an on-line platform (see Figure 1). The first is the Big-Five Inventory 10 (BFI-10) [40], a list of 10 questions aimed at measuring the personality in terms of the Big-Five traits, five broad dimensions known to capture most individual differences (see Section 4.3 for more details). The second is the Rahim Organizational Conflict Inventory II (ROCI-II) [39], a questionnaire aimed at measuring the Conflict Handling Style, i.e. the attitude of individuals in competitive discussions (see Section 4.4 for more details). All participants have accepted to fill the questionnaires and, therefore, the completion rate is $100 \%$.

The reason for administering the BFI-10 is that personality is the latent construct that accounts for "individuals' characteristic patterns of thought, emotion, and behavior together with the psychological mechanisms - hidden or not - behind those patterns" [17]. Thus, differences in personality might explain persuasiveness differences. The ROCIII was adopted because the scenario involves frequent disagreements and the questionnaire measures the behavioral strategy people tend to adopt when they are involved in competitive discussions [39]. Hence differences in Conflict Handling Style might explain why certain people convince their counterpart more often than others. Furthermore, both personality and conflict handling style were shown to influence negotiations (see Section 2.2).

The two questionnaires are administered and completed in the days before the experiments in order to attenuate as much as possible potential effects on the interaction outcomes. However, it is not possible to estimate demand char- acteristics or potential confounds that arise from having completed these questionnaires prior to participation in the negotiation. In particular, it is not possible to know whether filling the questionnaires changes attitude and behavior of the participants during the call.

\subsection{Experimental Protocol: the Call}

The day of the experiment, the participants of a call are invited to the University of Glasgow (School of Computing Science) and they perform all the steps of the following protocol (see Figure 1):

- Step 1: The two subjects are conducted in two separate rooms without encountering one another (see end of Section 3.1 for the assignment of the room).

- Step 2: Once in their room, the participants are asked to read the document in Appendix A, where they find the explanation of the task and the list of the 12 items at the core of the WST.

- Step 3: Before starting the call, the subjects fill the form available at the end of the document in Appendix A. Here, they must write a decision ("Yes" or "No") for each item of the list (left column of the form). This makes it possible to know, for each item, the decision made by each subject before any interaction with their counterpart. The subjects are asked to make a decision for every item (the call does not start if any item is left blank).

- Step 4: The two subjects receive a mobile phone (the same model for both participants).

- Step 5: One of the two subjects, selected randomly, calls the other with the mobile phone provided by the experimenters. 


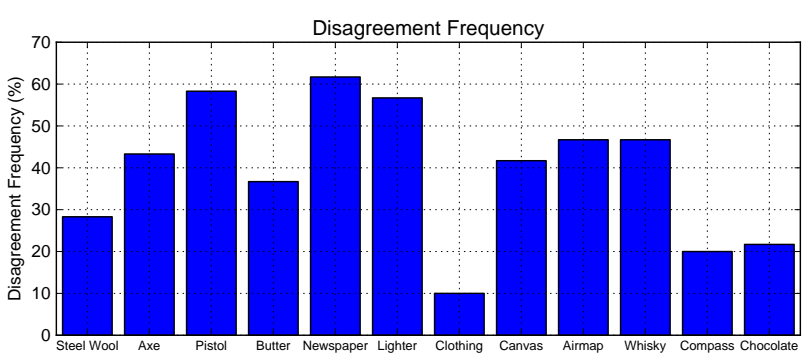

Fig. 2 The plot shows the percentage of calls where there was a disagreement on each item.

- Step 6: During the call, the subjects discuss item by item and negotiate a common solution ("Yes" or "No") that is the final outcome of the task (the decision is written on the right column of the form available at the end of the document in Appendix A). The call cannot be interrupted and cannot finish before a decision has been made for all items.

At the end of the call, the forms in Appendix A show not only what are the individual decisions of the subjects before the phone call, but also the final, consensual decision reached during the call. Thus, it is possible to know, for each item, whether the subjects agreed or disagreed before the call and, in the latter case, who is the subject that persuaded the other. Figure 2 shows, for each item, the percentage of calls where discussion was needed to reach a common decision. While some items (e.g., the clothing) were discussed only a few times, others were frequently debated between participants.

\section{Results}

The dataset includes 60 phone calls and, in each of them, the subjects must make 12 decisions (one per item). Thus, the total number of decisions made during the experiment is $60 \times 12=720$. In 437 cases $(60.7 \%$ of the total), the subjects have made the same decision when they performed the task individually before the call (third step of the protocol outlined above). In these cases, the subjects briefly discuss the item just to confirm their decision. In the remaining 283 cases $(39.3 \%$ of the total), the subjects have made different decisions before the call and, hence, they need to discuss in order to reach consensus. In these cases, one of the two subjects must necessarily persuade the other. The persuasiveness can be measured at two levels. At the item level, we consider the decision on each conflicting item as the variable of interest. We report the percentage of items where subjects of one category convince the others about their decision. At the call level, we aggregate the result for each call. The most persuasive subjects are those that convince the other in the largest number of items in the call.

\begin{tabular}{|l|c||c|c|}
\hline & Total & Item Level & Call Level \\
\hline Female & $63(52.5 \%)$ & $48.0 \%$ & $60.0 \%$ \\
\hline Male & $57(47.5 \%)$ & $52.0 \%$ & $40.0 \%$ \\
\hline
\end{tabular}

Table 2 Gender effects. The table reports the gender compositon of the subjects ("Total" column) as well as the persuasiveness of male and female subjects at both call and item level. According to a two-tailed binomial test, the $p$-value is higher than 0.42 for all persuasiveness figures.

The subjects get the same information (see Appendix A), undergo the same protocol (see Section 3.3), use the same phone model and, by scenario design, hold similar competences and skills about the WST. However, at the item level, the subjects that receive the call persuade those who make the call significantly more frequently than the other way around, namely $59.0 \%$ of the times ( $p$-value 0.003 according to a two-tailed binomial test). The effect is even more evident at the call level, the subjects receiving the call are the most persuasive in $70.0 \%$ of the 51 conversations that do not end with a tie ( $p$-value 0.005 according to a two-tailed binomial test).

According to the experimental protocols adopted in most negotiation studies [37, 43, 45, 47], each subject participates only in one call. Therefore, it is not possible to verify weather a change in role for the same subject (e.g., from caller to receiver) corresponds to a change of persuasiveness as well. However, this ensures that the subjects are not familiar with the task, a condition that eliminates effects and biases difficult to assess.

The results seem to be in line with the indications of Section 2.1 showing that the phones make it easier for noncooperative negotiation participants to maximize their individual profit (there is one winner in 51 calls out of the total 60). However, this does not explain why receivers should be more effective than callers. Therefore, it is necessary to verify whether the result is the effect of other factors known to make a difference in negotiations (age, gender, personality and conflict handling style).

\subsection{Gender Effects}

The data includes 31 calls where participants have different gender and the results of this section (see Table 2) apply to them. In these conversations, men persuade women $52.0 \%$ of the 125 times that consensus about an item must be reached through discussion ( $p$-value 0.72 according to a two-tailed binomial test). At the call level, men appear to be most persuasive in $60.0 \%$ of the cases, but the $p$-value of such an observation is 0.42 (according to a two-tailed binomial test), well above the acceptance level of 0.05 . In other words, gender effects, if any, are too moderate to produce observable effects on discussion outcomes and persuasive- 
ness. The result is in line with previous findings of the literature (see Section 2.2) showing that gender, overall, does not influence sigificantly the outcome of negotiations [44, 48]. Furthermore, the results show that gender cannot explain the persuasiveness difference between callers and receivers because men and women are evenly distributed across these two conditions. In other words, gender cannot be considered an explanation of the persuasiveness difference between callers and receivers observed in the experiments.

\subsection{Age Effects}

The upper plot of Figure 3 shows the age distribution of the experiment participants. The subjects are between 18 and 64 years old (average and standard deviation are 28.9 and 12.2, respectively). In 35 calls, the age difference is lower or equal to 10 years (see lower plot of Figure 3), but in the other cases the age difference goes up to 44 years. Since the subjects are unacquainted and do not meet before the call, it is probably difficult for them to estimate how old is their counterpart. However, the age difference might still have an effect on the negotiation outcomes.

At the item level, the younger participants of each call win in $54.1 \%$ of the cases ( $p$-value 0.999 according to a twotailed binomial test). At the call level, the percentage of successes for the younger participant is $51.0 \%$ ( $p$-value 0.190 according to a two-tailed binomial test). Therefore, age effects, if any, are too moderate to produce observable effects on the outcomes of the negotiation. Furthermore, in the 55 calls where the participants are of different age, the older subject is the receiver in $52.7 \%$ of the cases ( $p$-value 0.787 according to a two-tailed binomial test). In other words, older and younger subjects of each call are distributed evenly across callers and receivers. Therefore, the age difference cannot be considered an explanation for the higher persuasiveness of the receivers.

\subsection{Personality Effects}

Differences in personality might explain persuasiveness differences between the individuals that call and those that receive, especially if people with certain traits tend to be more frequent in one of the two conditions. For this reason, before the experiment, all subjects filled a personality questionnaire [40] based on the Big-Five model, a personality representation relying on five traits known to capture most of the individual differences:

- Extraversion: Active, Assertive, Energetic, etc.

- Agreeableness: Appreciative, Kind, Generous, etc.

- Conscientiousness: Efficient, Organized, Thorough, etc.

- Neuroticism: Anxious, Self-pitying, Tense, etc.
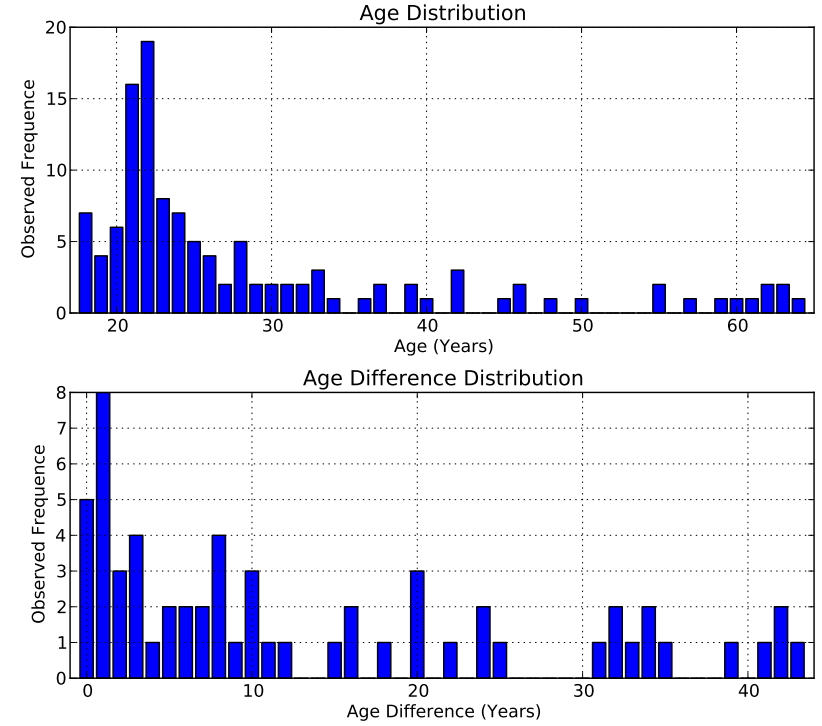

Fig. 3 The upper histogram shows the age distribution across the 120 participants, the lower histogram shows the age difference distribution across the 60 calls.

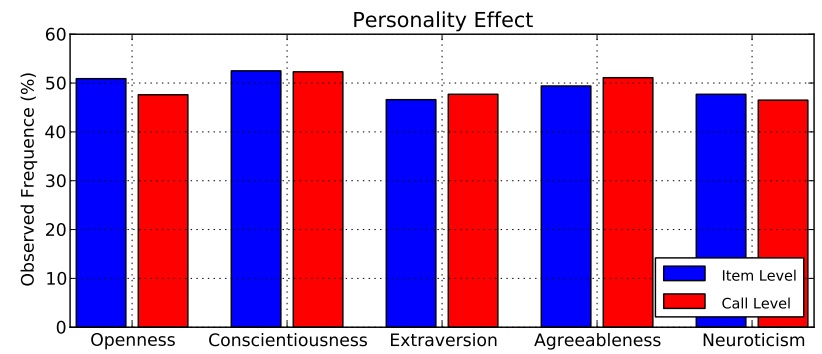

Fig. 4 The plot shows the percentage of times the subject with the higher score along a given trait is the most persuasive at the item and call level. The $p$-value is always above 0.3 (according to a two-tailed binomial test).

\section{- Openness: Artistic, Curious, Imaginative, etc.}

The analysis of the questionnaires provides five scores, one per trait, that account for how well the adjectives above describe the personality of each subject. If a trait influences the outcome of the discussions, the subjects that score higher along such a trait - in a pair of persons involved in the same call - should tend to persuade (or to be persuaded by) their interlocutor significantly more frequently. The plot of Figure 4 shows, for each trait, how frequently the dyad members with the higher trait score are more persuasive at item and call level. In all cases, the $p$-value is higher than 0.3 (according to a two-tailed binomial test). Hence, the effect of personality traits, if any, is too small to produce observable consequences in terms of persuasiveness. Furthermore, people that score higher on a given trait distribute evenly across callers and receivers and possible effects would still not explain the persusasiveness gap between the two conditions. 


\subsection{Conflict Handling Style Effects}

Conflict is a "mode of interaction [where] the attainment of the goal by one party precludes its attainment by the others" [23]. When there is disagreement, the two participants of each call start from opposite decisions, namely "Yes" and "No". Since the decision has to be consensual, if one of the two positions wins, the other necessarily loses. In other words, the subjects involved in one call pursue, in case of disagreement about an item, incompatible goals and are in conflict. For this reason, the subjects filled, before the experiment, a questionnaire about their conflict handling style, i.e. the behavioral strategy they tend to adopt when involved in competitive discussions [39]. In fact, the way subjects deal with conflict might influence the discussion outcomes and explain the persuasiveness differences observed in the experiment (see Section 2.2). The questionnaire provides five scores that measure how well the behavior of a subject matches one of the following tendencies:

- Avoiding: individuals tend to accept any, possibly unfavourable outcomes proposed by others to avoid conflict or unpleasant interaction.

- Compromising: individuals tend to find a trade-off between all, possibly incompatible outcomes proposed by different actors.

- Dominating: individuals tend to impose the outcomes they propose while rejecting those proposed by other actors.

- Integrating: individuals tend to find outcomes favourable and satisfactory for most, possibly all actors involved in the discussion.

- Obliging: individuals tend to address needs and suggestions by others at the cost of accepting, if necessary, unfavourable outcomes.

Figure 5 shows the percentage of times that, in a given discussion, the individuals with higher scores are more persuasive, at both item and call level. The only statistically significant effect ( $p$-value 0.035 according to a two-tayled binomial test) is that the most "integrating" subjects of each pair persuade their interlocutor $57.0 \%$ of the times at the item level (in line with the findings in [11]). However, these subjects appear the same number of times among both those who call and those who are called. Therefore, the effect does not explain the difference in persuasiveness between callers and receivers. Furthermore, the effect of the integrating strategy is not statistically significant at the call level ( $p$-value $=0.14$ according to a two-tayled binomial test). In this case as well, the conflict handling style does not produce effects that can explain the persuasiveness difference between subjects that call and subjects that are called.

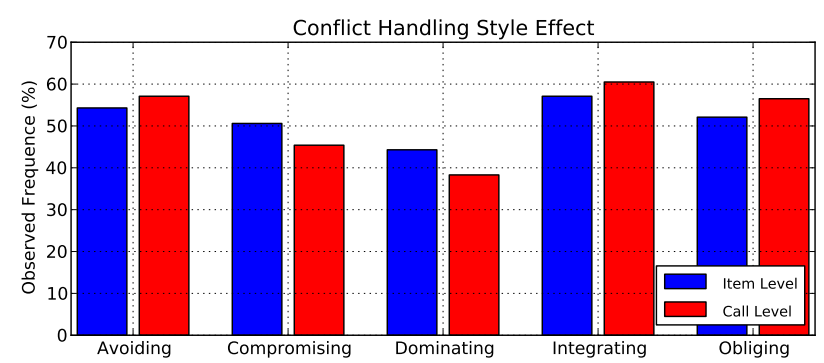

Fig. 5 The plot shows the percentage of times the subject with the higher score along a given conflict handling style is the most persuasive at the item and call level. The only case where the $p$-value is below 0.005 , according to a two tayled binomial test, is the integrating style at the item level.

\section{Conclusions}

This paper investigates the impact of mobile phones on the outcome of negotiations between unacquainted individuals. In particular, the paper has confirmed that phones, far from being passive communication media, influence the social interplay of their users and, potentially, can induce measurable behavioral changes. In the scenario adopted for the experiments of this work, calling or being called appears to make a significant difference in terms of persuasiveness. Gender, age, personality and conflict handling style were considered as an alternative explanation, but they appear to have negligible influence on the outcomes of the experiment. Calling or being called, remains the factor that better explains the persuasiveness differences observed among subjects.

The lack of symmetry between callers and receivers was previously observed in [16], where callers were shown to have an advantage in terms of setting the call agenda, defining the tone of the conversation, etc. The results of this work seem to be in contradiction (the receivers appear to be in a more favorable condition). However, this might simply depend on the fact that the experiments take place in a controlled setting where both participants share the same information about the call. Furthermore, the receivers know that they are going to be called and, therefore, there is no surprise effect, something that seems to play a role in the findings of [16].

Unlike previous research on interplay between communication media and negotiation (see Section 2), this work focused on mobile phones and not on landline ones. The reason is that the number of individuals that subscribe only to mobile telephony services is constantly increasing and the trend is likely to continue in the foreseeable future [21]. However, both types of phone have the same richness (see Section 2.1) and the findings of this work seem to be in line with those obtained over landline phones under at least two important points of view. The first is that both media are efficient, i.e. they allow one to exchange sufficient information to complete the negotiation task [30]. No breakdowns have 
been observed like it happens, e.g., using text only chat or written documents. The second is that, via phone, the maximization of the individual profit (the number of won items in this work) tends to be more frequent than the maximization of the joint profit [43]. Still, nothing conclusive can be said about the comparison between mobile and landline phones without repeating the experiment with these latter.

To the best of our knowledge, this is one of the few works that measure the effect of mobile phones on one-toone conversations. Unlike other works in the literature (see Section 2), this article does not propose software applications expected to change the behavior of people when installed on their phones. The experiments of this work simply show that the very use of mobile phones results into a change of persuasiveness. The results seem to be in line with the indications of the literature about the effect of communication media on negotiations (see above). However, the experiments were perfomed in a controlled setting and it is unclear how much they can be generalized. Hence, future work will aim in particular at investigating whether different, more naturalistic conditions lead to similar findings.

Acknowledgements This work was supported in part by the European Commission through the FP7 Network of Excellence SSPNet (GA 241287), in part by the Swiss National Science Foundation via the National Centre for Competence in Research IM2 (Interactive Multimodal Informationa Management), and in part by the Fnnish Ministry for Technological Innovation via the HEI project.

\section{References}

1. Aarts H, Custers R, Marien H (2008) Preparing and Motivating Behavior Outside of Awareness. Science 319(5870):1639-1639

2. Abraham C, Michie S (2008) A Taxonomy of Behavior Change Techniques Used in Interventions. Health Psychology 27(3):379-387

3. Aharony N, Pan W, Ip C, Khayal I, Pentland A (2011) Social fMRI: Investigating and shaping social mechanisms in the real world. Pervasive and Mobile Computing 7(6):643-659

4. Arminen I, Weilenmann A (2009) Mobile Presence and Intimacy - Reshaping Social Actions in Mobile Contextual Configuration. Journal of Pragmatics 41(10):19051923

5. Barry B, Friedman R (1998) Bargainer characteristics in distributive and integrative negotiation. Journal of Personality and Social Psychology 74(2):345-359

6. Bazerman M, Curhan J, Moore D, Valley K (2000) Negotiation. Annual Review of Psychology 51:279-314

7. Bradner E, Mark G (2002) Why distance matters: effects on cooperation, persuasion and deception. In: Pro- ceedings of the ACM International Conference on Computer Supported Cooperative Work, pp 226-235

8. Cardy E (2005) An experimental field study of the GOTV and persuasion effects of partisan direct mail and phone calls. The Annals of the American Academy of Political and Social Science 601(1):28-40

9. Carnevale P, Pruitt D (1992) Negotiation and mediation. Annual Review of Psychology 43:531-582

10. Chambless D, Ollendick T (2001) Empirically Supported Psychological Interventions: Controversies and Evidence. Annual Review of Psychology 52:685-716

11. Cheung S, Yiu T, Yeung S (2006) A study of styles and outcomes in construction dispute negotiation. Journal of Construction Engineering and Management 132(8):805-814

12. Chiu MC, Chang SP, Chang YC, Chu HH, Chen $\mathrm{CCH}$, Hsiao FH, Ko JC (2009) Playful bottle: a mobile social persuasion system to motivate healthy water intake. In: Proceedings of the International Conference on Ubiquitous Computing, pp 185-194

13. Dourish P, Bell G (2011) Divining a digital future: mess and mythology in ubiquitous computing. MIT Press

14. Durham C, Locke E, Poon J, McLeod P (2000) Effects of group goals and time pressure on group efficacy, information-seeking strategy, and performance. Human Performance 13(2):115-138

15. Fogg B (2002) Persuasive technology: using computers to change what we think and do. Ubiquity 2002(December):5

16. Fortunati L (1995) Gli italiani al telefono. Franco Angeli

17. Funder D (2001) Personality. Annual Review of Psychology 52:197-221

18. Gasser R, Brodbeck D, Degen M, Luthiger J, Wyss R, Reichlin S (2006) Persuasiveness of a mobile lifestyle coaching application using social facilitation. In: IJsselsteijn W, Kort A, Midden C, Eggen B, Hoven E (eds) Persuasive Technology, Lecture Notes in Computer Science, vol 3962, pp 27-38

19. Harper R (2010) Texture. MIT Press

20. ITU (2010) The world in 2010: ICT facts and figures. Tech. rep., International Telecommunication Union

21. ITU (2013) Measuring the information society. Tech. rep., International Telecommunication Union

22. Joshi M, Davis E, Kathuria R, Weidner C (2005) Experiential learning process: Exploring teaching and learning of strategic management framework through the winter survival exercise. Journal of Management Education 29(5):672-695

23. Judd C (1978) Cognitive effects of attitude conflict resolution. Journal of Conflict Resolution 22(3):483-498

24. Kalba K (2008) The Global Adoption and Diffusion of Mobile Phones. Tech. Rep. December, Center for Infor- 
mation Policy Research Harvard University

25. Kray L, Reb J, Galinsky A, Thompson L (2004) Stereotype reactance at the bargaining table: The effect of stereotype activation and power on claiming and creating value. Personality and Social Psychology Bulletin 30(4):399-411

26. Lathia N, Pejovic V, Rachuri K, Mascolo C, Musolesi M, Rentfrow P (2013) Smartphones for large-scale behaviour change interventions. IEEE Pervasive Computing 12(3):66-73

27. Ling R (2008) New Tech, New Ties. How Mobile Communication is Reshaping Social Cohesion. MIT Press

28. Madan A, Cebrian M, Moturu S, Farrahi K, Pentland A (2012) Sensing the "Health State" of our Society. IEEE Pervasive Computing (to appear)

29. Magee J, Galinsky A, Gruenfeld D (2007) Power, propensity to negotiate, and moving first in competitive interactions. Personality and Social Psychology Bulletin 33(2):200-212

30. McGinn K, Croson R (2004) What do communication media mean for negotiations? A question of social awareness. In: Gelfand M, Brett J (eds) The handbook of negotiation and culture, Stanford University Press, pp 334-339

31. Miner F (1984) Group versus individual decision making: An investigation of performance measures, decision strategies, and process losses/gains. Organizational Behavior and Human Performance 33(1):112-124

32. Mohammadi G, Park S, Sagae K, Vinciarelli A, Morency LP (2013) Who is persuasive? The role of perceived personality and communication modality in social multimedia. In: Proceedings of the ACM International Conference on Multimodal Interaction (to be presented)

33. Nass C, Brave S (2005) Wired for speech: How voice activates and advances the Human-Computer relationship. The MIT Press

34. Nass C, Min Lee K (2001) Does computer-synthesized speech manifest personality? experimental tests of recognition, similarity-attraction and consistencyattraction. in Journal of Experimental Psychology: Applied 7(3):171-181

35. Pianesi F, Zancanaro M, Lepri B, Cappelletti A (2007) A multimodal annotated corpus of consensus decision making meetings. Language Resources and Evaluation 41(3-4):409-429

36. Pinkley R, Northcraft G (1994) Conflict frames of reference: Implications for dispute processes and outcomes. Academy of Management Journal 37(1):193-205

37. Purdy J, Nye P, Balakrishnan P (2000) The impact of communication media on negotiation outcomes. International Journal of Conflict Management 11(2):162187
38. Raento M, Oulasvirta A, Eagle N (2009) Smartphones: an Emerging Tool for Social Scientists. Sociological Methods \& Research 37(3):426-454

39. Rahim M (1983) A Measure of Styles of Handling Interpersonal Conflict. The Academy Management Journal 26(2):368-376

40. Rammstedt B, John O (2007) Measuring Personality in One Minute or Less: A 10-item Short Version of the Big Five Inventory in English and German. Journal of Research in Personality 41(1):203-212

41. Reeves B, Nass C (1996) The media equation: How people treat computers, television, and new media like real people and places. Cambridge University Press

42. Rogelberg S, Rumery S (1996) Gender diversity, team decision quality, time on task, and interpersonal cohesion. Small Group Research 27(1):79-90

43. Sheffield J (1995) The effect of communication medium on negotiation performance. Group Decision and Negotiation 4(2):159-179

44. Stuhlmacher A, Walters A (1999) Gender differences in negotiation outcome: A meta-analysis. Personnel Psychology 52(3):653-677

45. Suh K (1999) Impact of communication medium on task performance and satisfaction: an examination of media-richness theory. Information \& Management 35(5):295-312

46. Thompson L, Wang J, Gunia B (2010) Negotiation. Annual Review of Psychology 61:491-515

47. Valley K, Moag J, Bazerman M (1998) "a matter of trust": Effects of communication on the efficiency and distribution of outcomes. Journal of Economic Behavior \& Organization 34(2):211-238

48. Walters A, Stuhlmacher A, Meyer L (1998) Gender and negotiator competitiveness: A meta-analysis. Organizational Behavior and Human Decision Processes 76(1):1-29

49. Wilson T (2006) The Power of Social Psychological Interventions. Science 313(5791):1251-1252

\section{Appendix A}

\section{THE SCENARIO}

You are member of a rescue team. Your duty is to provide assistance to any person facing dangerous situations in a large area of Northern Canada. You have just received an SOS call from a group of people that survived a plane crash and report on their situation as follows: "Both the pilot and co-pilot were killed in the crash. The temperature is $25^{\circ} \mathrm{C}$, and the night-time temperature is expected to be $-40^{\circ} \mathrm{C}$. There is snow on the ground, and the countryside is wooded with several rivers crisscrossing the area. The nearest town is $32.2 \mathrm{~km}$ ( $\sim 20$ miles) away. We are all dressed in city clothes appropriate for a business meeting." The survivors have managed to extract 12 objects from the plane. But they have to leave the site of the accident, carrying only a few objects - the less the better - in order to increase their chances of survival. 


\section{THE MISSION}

Your mission is to identify the objects most likely to maximize the chances of survival of the plane passengers. The protocol includes two steps:

- Step 1 - Individual Step

You receive a table (p.3) showing the 12 items and you have to decide for each one of them whether it is worth carrying or not. You must write your decision, using YES or NO (YES: they have to carry it, NO: must not carry it), in the column on the left of the table.

- Step 2 - Discussion

You will have a telephone conversation with another member of the rescue team in order to decide together which objects must be carried and what objects must be left in the plane.

As the call is a matter of life and death for the survivors, you will follow an emergency discussion protocol:

1. Consider the first object in the list.

2. Discuss with your colleague whether or not the object must be carried until you make a decision. The decision must be consensual and you can take as much time as you need in order to make the right decision.

3. Write your decision in the column to the right part of the table (p.3): the decision must be the same for both participants.

4. Once you have made a decision, move to the following object and repeat steps 2 and 3 .

5. Continue until all objects have been considered and a consensual decision has been made for each one of them.

Please consider the following:

- Discuss one object at a time and move onto the next only after a consensual decision has been made.

- Once a decision has been made, do not go back and change the decision about previous objects. Discuss the objects in the order shown on the attached list.

- Do not interrupt the call until all objects have been discussed and all decisions have been made.

At the end of the conversation you have to return the table with the items, completed with "YES" or "NO" decisions for each item. The results must be the same for both you and your colleague. The phone call will be recorded.

\section{A REWARDING SCHEME}

You will receive $£ 6$ for your participation, but you can significantly increase your reward if you make the right decisions. Some objects are actually necessary and must be carried while others should be left on the crash site:

- You receive $£ 3$ extra, each time you decide to carry an item that must actually be carried (a right item).

- You lose $£ 3$, each time you decide to carry an item that must not actually be carried.

- You lose $£ 3$ for each decision marked on your list that is different from the one of your colleague.

In any case, a payment of $£ 6$ is guaranteed for your participation.

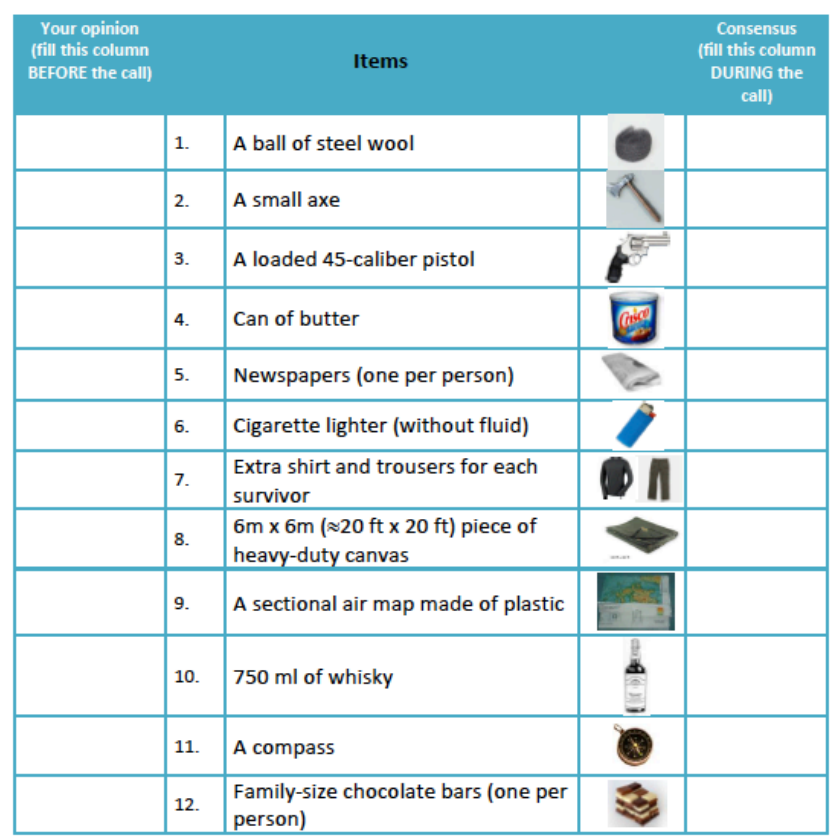

